\title{
Synergistic Uric Acid-Lowering Effects of the Combination of Chrysanthemum indicum Linne Flower and Cinnamomum cassia (L.) J. Persl Bark Extracts
}

\author{
Young-Sil Lee, ${ }^{1}$ Eunjung Son, ${ }^{1}$ Seung-Hyung Kim, ${ }^{2}$ \\ Yun Mi Lee, ${ }^{1}$ Ohn Soon Kim, ${ }^{1}$ and Dong-Seon Kim ${ }^{1}$ \\ ${ }^{1}$ KM Convergence Research Division, Korea Institute of Oriental Medicine, 1672 Yuseong-daero, \\ Yuseong-gu, Daejeon 34054, Republic of Korea \\ ${ }^{2}$ Institute of Traditional Medicine and Bioscience, Daejeon University, 62 Daehak-ro, Dong-gu, Daejeon 34520, Republic of Korea \\ Correspondence should be addressed to Dong-Seon Kim; dskim@kiom.re.kr
}

Received 9 February 2017; Revised 18 April 2017; Accepted 22 May 2017; Published 9 July 2017

Academic Editor: Attila Hunyadi

Copyright (C) 2017 Young-Sil Lee et al. This is an open access article distributed under the Creative Commons Attribution License, which permits unrestricted use, distribution, and reproduction in any medium, provided the original work is properly cited.

\begin{abstract}
Chrysanthemum indicum Linne flower (CF) and Cinnamomum cassia (L.) J. Persl bark (CB) extracts have served as the main ingredients in several prescriptions designed to treat hyperuricemia and gout in traditional Chinese and Korean medicine. However, little is known about the combination effects of a CF and CB (CC) mixture on hyperuricemia. In our study, we investigated the antihyperuricemic effects of CC mixture and the mechanisms underlying these effects in normal and potassium oxonate- (PO-) induced hyperuricemic rats. The CC mixture significantly decreased uric acid levels in normal and PO-induced hyperuricemic rats and showed the enhanced hypouricemic effect compared to CF or CB alone. Furthermore, the CC mixture increased renal uric acid excretion in PO-induced hyperuricemic rat. We found that CC mixture and its major components, chlorogenic acid, 3,4-dicaffeoylquinic acid (isochlorogenic acid), coumarin, cinnamaldehyde, trans-cinnamic acid, and o-methoxycinnamaldehyde, inhibit the activity of xanthine oxidase (XOD) in vitro. The CC mixture exerts antihyperuricemic effects accompanied partially by XOD activity inhibition. Therefore, the CC mixture may have potential as a treatment for hyperuricemia and gout.
\end{abstract}

\section{Introduction}

Hyperuricemia is a metabolic disease characterized by elevated blood uric acid levels [1], the prevalence of which has increased worldwide. Hyperuricemia results from increased production or impaired excretion of uric acid $[2,3]$, and elevated uric acid levels cause accumulation of urate crystals in joints and the kidneys, leading to gout and gouty arthritis $[4,5]$. It was also recently shown that hyperuricemia is associated with hyperlipidemia, hypertension, cardiovascular diseases, and diabetes [6]. Therefore, uric acid level regulation may play an important role in the prevention and treatment of various diseases, including hyperuricemia.

Uric acid is a final product of purine catabolism and is produced via the catalytic activities of xanthine oxidase (XOD), a rate-limiting enzyme responsible for converting hypoxanthine to xanthine, which is subsequently converted to uric acid [7]. In humans, seventy percent of the uric acid in the body is excreted on a daily basis by urate transporters, including the urate-anion transporter and organic anion transporter in the kidneys [8]. Accordingly, inhibiting uric acid biosynthesis and increasing uric acid excretion may be useful therapeutic approaches for the treatment of hyperuricemia. Currently, XOD inhibitors (allopurinol (AP) and febuxostat) and uricosuric agents (benzbromarone and probenecid) are used as antihyperuricemia drugs in clinical practice [9]. However, allopurinol and benzbromarone are poorly tolerated and reportedly induce side effects, such as drug allergies, renal failure, and hypersensitivity syndrome. These adverse effects limit the use of these drugs [10-13]. Thus, new antihyperuricemia agents are needed. Currently, natural products, such as herbal medicines, have emerged as 
new therapies that can overcome these limitations [13] and the numbers of studies regarding traditional herbal plants are increasing.

To identify herbal plants with antihyperuricemic effects, we screened hundreds of herbal medicinal plant extracts and found that Chrysanthemum indicum Linne flower and Cinnamomum cassia (L.) J. Persl bark extracts exert uric acidlowering effects in animal models in a preliminary study. Chrysanthemum indicum is a traditional medicinal plant that has been used to treat inflammation-related diseases, several infectious diseases, and hypertension in Korea and China. Additionally, its flowers are used as herbal teas for the treatment of eye diseases and headaches and have various biological properties, including antioxidation and antitumor properties $[14,15]$. Cinnamomum cassia bark, which is also known as cinnamomi cortex, has been used in traditional medicine to treat immune-related diseases, gastritis, diarrhea, and cancer and is also a popular spice worldwide $[16,17]$. Cinnamomum cassia extracts also exhibit anticancer and antidiabetic effects $[18,19]$. It has been reported that C. indicum flower and C. cassia bark have served as the main ingredients in several prescriptions designed to treat hyperuricemia and gout in traditional Chinese medicine [20]. According to a recent report, in an in vitro study of traditional Chinese medicinal plants, methanol extracts of C. indicum flower and C. cassia bark inhibited XOD activity [21]. Oil extracts from C. indicum flower, C. cassia bark, and C. osmophloeum leaves reduced serum uric acid levels in potassium oxonate- (PO-) induced hyperuricemic animals $[20,22,23]$. These findings indicate that C. indicum flower and $C$. cassia bark have antihyperuricemic effects. However, the combined effects of $C$. indicum flower and C. cassia bark extracts have not been studied. Thus, in the present study, we investigated the antihyperuricemic effects of $C$. indicum Linne flower, C. cassia (L.) J. Persl bark, and their combination in $\mathrm{PO}$-induced hyperuricemic rats and normal rats, as well as the mechanism underlying these effects.

\section{Materials and Methods}

2.1. Materials. Chlorogenic acid, coumarin, 3,4-dicaffeoylquinic acid, cinnamaldehyde, trans-cinnamic acid, and $o$ methoxycinnamaldehyde were purchased from ChemFaces (China), respectively. The purities of them were determined to be $98 \%$ by high performance liquid chromatography (HPLC) analysis. Acetonitrile, methanol, and water were purchased from J. T. Baker (Phillipsburg, NJ, USA). Trifluoroacetic acid, allopurinol, dimethyl sulfoxide, xanthine oxidase from bovine milk (0.09 units/mg solid), xanthine sodium salt, and phosphate buffer ( $\mathrm{pH} 7.2)$ were obtained from Sigma-Aldrich (St. Louis, MO, USA).

2.2. Sample Preparation. Flowers of $C$. indicum Linne and barks of C. cassia (L.) J. Persl were purchased from Kwangmyungdang Medical Herbs (Ulsan, South Korea). Flowers of C. indicum Linne, called "Indian Dendranthema" in English, were obtained at Zhejiang, China, and barks of C. cassia (L.) J. Persl, called "cinnamon" in English, were obtained at Yen Bai,
Vietnam, respectively. C. indicum flower (CF) and C. cassia bark (CB) were identified by the Classification and Identification Committee of the Korea Institute of Oriental Medicine (KIOM) and their voucher specimens (numbers GHP-030 and 078) were stored at the herbarium of the Department of Herbal Resources Research of KIOM. They were ground and refluxed with $70 \%(v / v)$ ethanol for 3 hours using a Soxhlet extractor. Filtered extracts were concentrated using an evaporator (EYELA, Tokyo Rikakikai, Tokyo, Japan) at $45^{\circ} \mathrm{C}$ and then dried with a freeze dryer (Il-Sin Programmable Freeze Dryer-20R, Dongducheon, Korea) for 3 days. A total of $35.2 \mathrm{~g}$ of extract was obtained from $400 \mathrm{~g}$ of dried CF, and $31.0 \mathrm{~g}$ of extract was obtained from $400 \mathrm{~g}$ of dried $\mathrm{CB}$ for yields of 8.82 and $7.76 \%$, respectively. Mixed samples were prepared from combinations of $\mathrm{CF}$ and $\mathrm{CB}$ extracts at weight ratios of $1: 1,1: 2,1: 4,2: 1$, and $4: 1$ to yield the following mixtures: CC11, CC12, CC14, CC21, and CC41. The samples were dissolved in $100 \%$ dimethyl sulfoxide to a final concentration of $50 \mathrm{mg} / \mathrm{ml}$ for in vitro efficacy testing and dissolved in methanol to a final concentration of $10 \mathrm{mg} / \mathrm{ml}$ for HPLC analysis. Each sample subjected to HPLC analysis was filtered using $0.45 \mu \mathrm{m}$ syringe filters (Millipore, Billerica, MA, USA).

2.3. Animals. Male SD rats (7 weeks) were purchased from Orient Bio (Seongnam, Korea) and were maintained at a temperature of $22 \pm 1^{\circ} \mathrm{C}$ in a $50 \pm 10 \%$ humidity-controlled room under a $12 \mathrm{~h}$ light $/ 12 \mathrm{~h}$ dark cycle. The rats were allowed free access to a laboratory diet and water. The experimental design was approved by the Committee on Animal Care of the $\mathrm{KIOM}$, and all experiments were performed in accordance with committee guidelines.

2.4. Sample Treatment in Normal Rats. The rats were randomized into the following 9 groups ( $n=8$ /group) based on body weight: (1) a normal control group (NC), (2) a CF group, (3) a CB group, (4) a CC11 group, (5) a CC12 group, (6) a CC14 group, (7) a CC21 group, (8) a CC41 group, and (9) an AP group. The rats of NC group were treated with vehicle (0.5\% CMC solution). CF, CB, and various CC mixtures in $0.5 \% \mathrm{CMC}$ solution were given to the rats via oral gavage at a dose of $200 \mathrm{mg} / \mathrm{kg}$. Allopurinol in $0.5 \%$ CMC solution was administered at a dose of $10 \mathrm{mg} / \mathrm{kg}$.

2.5. Hyperuricemia Induction and Sample Treatment. For hyperuricemia induction, the uricase inhibitor potassium oxonate (PO) was administered as previously described [24, 25], with slight modifications. For the experiment regarding the effects of $\mathrm{CF}, \mathrm{CB}$, and various $\mathrm{CC}$ mixtures, the rats were divided into the following 10 groups ( $n=8$ /group) based on body weight: (1) a normal control group (NC), (2) a hyperuricemia control group (PO), (3) a PO + CF group, (4) a $\mathrm{PO}+\mathrm{CB}$ group, (5) a PO + CC11 group, (6) a PO + CC12 group, (7) a $\mathrm{PO}+\mathrm{CC} 14$ group, (8) a $\mathrm{PO}+\mathrm{CC} 21$ group, (9) a PO + CC41 group, and (10) a PO + AP group. The rats in groups (2)-(10) were injected intraperitoneally with $250 \mathrm{mg} / \mathrm{kg}$ PO prepared in $0.5 \%$ CMC with $0.1 \mathrm{M}$ sodium acetate ( $\mathrm{pH} 5.0)$ to induce hyperuricemia except group (1) 


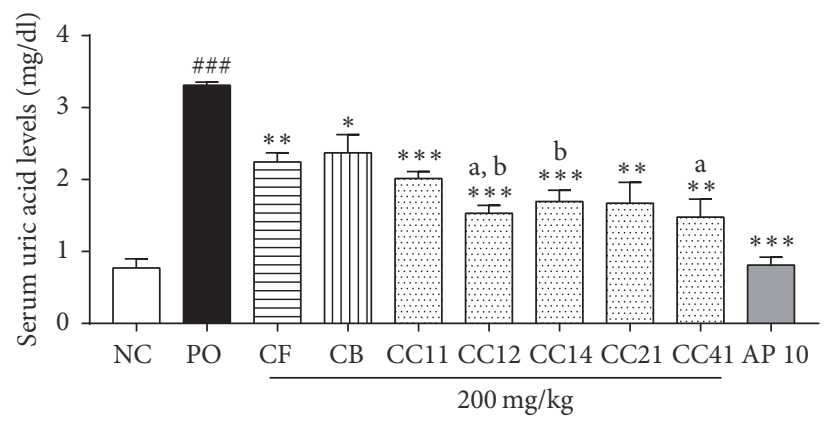

(a)

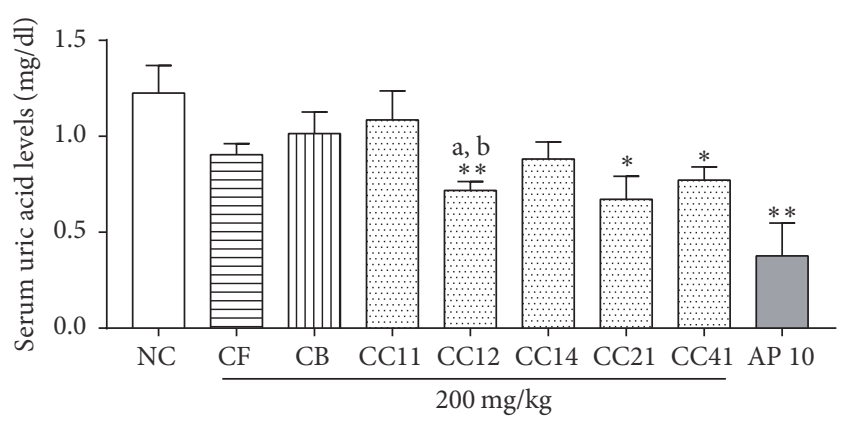

(b)

FIGURE 1: Effects of CB and CF and their combination on serum uric acid levels in PO-induced hyperuricemic rats (a) and normal rats (b). NC, normal control group; PO, potassium oxonate-induced hyperuricemia group; CF, $200 \mathrm{mg} / \mathrm{kg}$ Chrysanthemum indicum Linne flower ethanol extract; CB, $200 \mathrm{mg} / \mathrm{kg}$ Cinnamomum cassia (L.) J. Persl bark ethanol extract; CC, mixture of CF and CB; AP 10, $10 \mathrm{mg} / \mathrm{kg}$ allopurinol. Data are expressed as the mean $\pm \operatorname{SEM}(n=8) .{ }^{\# \# \#} p<0.001$ versus the NC group; ${ }^{*} p<0.05,{ }^{* *} p<0.01$, and ${ }^{* * *} p<0.001$ versus the PO group; ${ }^{\mathrm{a}} p<0.05$ versus the CF group; ${ }^{\mathrm{b}} p<0.05$ versus the CF group.

that received $0.5 \% \mathrm{CMC}$ with $0.1 \mathrm{M}$ sodium acetate. Groups (1) and (2) received vehicle $(0.5 \% \mathrm{CMC})$ by oral gavage, and groups (3)-(9) received CF, CB, CC11, CC12, CC14, CC21, and CC41 at a dose of $200 \mathrm{mg} / \mathrm{kg}$. Group (10) was treated with allopurinol at a dose of $10 \mathrm{mg} / \mathrm{kg}$. For the experiment examining the dose-dependent effects of the CC12 mixture, the rats were divided into the following 6 groups: (1) an NC group, (2) a PO group, (3) a $\mathrm{PO}+100 \mathrm{mg} / \mathrm{kg} \mathrm{CC} 12$ group, (4) a PO + $200 \mathrm{mg} / \mathrm{kg}$ CC12 group, (5) a PO + $400 \mathrm{mg} / \mathrm{kg}$ $\mathrm{CC1} 2$ group, and (6) a PO + AP group. All test samples were administered orally $30 \mathrm{~min}$ prior to PO injection.

2.6. Analysis of Uric Acid and Creatinine Levels in Serum and Urine. Urine samples were collected during $2 \mathrm{~h}$ after the final administration experiment and centrifuged $(3000 \mathrm{~g}$, $10 \mathrm{~min}, 4^{\circ} \mathrm{C}$ ) to remove particulate contaminants, and the supernatants were stored at $-80^{\circ} \mathrm{C}$ until analysis. Blood samples were collected via cardiac puncture under anesthesia $2 \mathrm{~h}$ after urine collection following PO injection. Serum was obtained via centrifugation at $3000 \mathrm{~g}$ for $10 \mathrm{~min}$ at $4^{\circ} \mathrm{C}$ after allowing the blood samples to clot for $2 \mathrm{~h}$ at room temperature. The separated serum was stored at $-80^{\circ} \mathrm{C}$ until analysis. Serum and urine uric acid (SUA and UUA, resp.) levels were determined by an enzymatic-colorimetric method using commercial assay kits (Biovision, USA) according to manufacturer's protocols. Serum and urine creatinine levels were analyzed by an autoanalyzer (Hitachi-7020, Hitachi Medical, Japan). Fractional excretion of uric acid (FEUA) was calculated by the formula $\operatorname{FEUA}(\%)=$ (urine uric acid levels $[\mathrm{mg} / \mathrm{dl}] \times$ Serum Creatinine levels $[\mathrm{mg} / \mathrm{dl}]) /($ serum uric acid levels $[\mathrm{mg} / \mathrm{dl}] \times$ urine creatinine levels $[\mathrm{mg} / \mathrm{dl}]) \times 100$.

2.7. Instrument Conditions for HPLC Analysis. To analyze the extracts, Agilent 1200 series instruments, including a degasser, binary pump, autosampler, and photodiode array detector, were used. Chromatographic separation was performed on a binary solvent manager, sample manager, column heater, and photodiode array detector with a Thermo
Acclaim $^{\mathrm{TM}}$ Polar Advantage C18 column $(4.6 \times 250 \mathrm{~mm}, 5 \mu \mathrm{m})$ at a flow rate of $1.5 \mathrm{ml} / \mathrm{min}$. The column oven was set at $40^{\circ} \mathrm{C}$. The mobile phase consisted of $0.1 \%$ trifluoroacetic acid buffer (A) and acetonitrile (B). The gradient elution conditions were as follows: $10-10 \% \mathrm{~B}$ for $0-5 \mathrm{~min}, 10-23 \% \mathrm{~B}$ for $5-20 \mathrm{~min}$, $23-25 \%$ B for $20-30 \mathrm{~min}, 25-25 \%$ B for $30-50 \mathrm{~min}, 25-50 \%$ B for $50-65 \mathrm{~min}, 50-100 \% \mathrm{~B}$ for $65-70 \mathrm{~min}, 100-100 \% \mathrm{~B}$ for $70-75 \mathrm{~min}, 100-10 \% \mathrm{~B}$ for $75-80 \mathrm{~min}$, and $10-10 \% \mathrm{~B}$ for $80-85 \mathrm{~min}$. The final sample consisted of $10 \mu \mathrm{l}$ of each sample, and the monitoring detector was set at a wavelength of $255 \mathrm{~nm}$.

2.8. In Vitro XOD Inhibition Assay. Inhibition of XOD activity was analyzed based on decreases in uric acid formation at $295 \mathrm{~nm}$ at $37^{\circ} \mathrm{C}$ using a spectrophotometer. The reaction mixture comprised $50 \mathrm{mM}$ sodium phosphate buffer ( $\mathrm{pH} 7.6$ ), $17.9 \mathrm{nM}$ xanthine sodium salt, and 0.04 units of xanthine oxidase, and the reaction was started by adding xanthine oxidase and xanthine. Test samples were dissolved in DMSO (final concentration $0.1 \%, \mathrm{v} / \mathrm{v}$ ) to final concentrations appropriate for the tested dose. XOD activity was compared with that of allopurinol, which served as a positive control. All the experiments were performed in triplicate, and $\mathrm{IC}_{50}$ values are expressed as the means of three experiments [26].

2.9. Statistical Analysis. Data are presented as the mean \pm SEM. Differences among the treatment groups were analyzed by one-way ANOVA and Dunnett's multiple comparison test was applied to identify the significance using Prism 7.0 software (GraphPad Software Inc., San Diego, CA, USA), and $p<0.05$ was considered statistically significant.

\section{Results}

3.1. Effects of CF, CB, and Various CC Mixtures on Serum Uric Acid Levels in PO-Induced Hyperuricemic Rats and Normal Rats. The effects of $\mathrm{CF}, \mathrm{CB}$, and various $\mathrm{CC}$ mixtures on serum uric acid levels in $\mathrm{PO}$-induced hyperuricemic rats and normal rats are shown in Figure 1. Serum uric acid levels 
in the PO group were significantly higher than those in the NC group $(p<0.001)$. Treatment with CF and CB at a dose of $200 \mathrm{mg} / \mathrm{kg}$ significantly reduced serum uric acid levels by $32 \%$ and $28 \%$, respectively, compared with the PO group ( $p<0.01, p<0.05)$. Additionally, administration of various $\mathrm{CC}$ mixtures (CC11, CC12, CC14, CC21, and CC41) at a dose of $200 \mathrm{mg} / \mathrm{kg}$ significantly reduced serum uric acid levels by $45-55 \%$ compared with the PO group $(p<0.001$, $p<0.001, p<0.001, p<0.01$, and $p<0.01$, resp., Figure $1(\mathrm{a})$ ), and $10 \mathrm{mg} / \mathrm{kg}$ AP reduced serum uric acid levels by $75 \%(p<0.001$, Figure $1(\mathrm{a}))$ and exhibited hypouricemic effects similar to those noted in the NC group. Moreover, treatment of CC12 and CC41 mixtures significantly reduced the serum uric acid levels compared with the CF group and CC12 and CC14 mixtures showed lower serum uric acid levels compared with the $\mathrm{CB}$ group in $\mathrm{PO}$-induced hyperuricemic rats $(p<0.05$, all). As shown in Figure 1(b), CF and CB treatment decreased serum uric acid levels in normal rats, although this effect was not significant. Three 3 CC mixtures, CC12, CC21, and CC41, significantly decreased serum uric acid levels by $42 \%, 45 \%$, and $40 \%$, respectively, compared with the NC group $(p<0.01, p<0.05$, and $p<0.05$, resp.), while the CC11 and CC14 mixtures had no effect on serum uric acid levels. Treatment with AP decreased serum uric acid levels by $70 \%(p<0.01)$ compared with the NC group. CC12 mixture reduced the serum uric acid levels compared with the $\mathrm{CF}$ and $\mathrm{CB}$ groups in normal rats $(p<0.05)$. In this study, we found that $\mathrm{CC}$ mixtures exert better antihyperuricemic effects than $\mathrm{CF}$ or $\mathrm{CB}$ alone and that the $\mathrm{CC} 12$ mixture elicited a $55 \%$ reduction in uric acid levels, which was greater than that induced by the other CC mixtures. Thus, we selected the CC12 mixture for further study.

\subsection{Dose-Dependent Effects of the CC12 Mixture on Serum} and Urine Uric Acid Levels in PO-Induced Hyperuricemic Rats. Serum uric acid levels were significantly higher in the PO group than in the NC group $(p<0.05)$. Treatment with the CC12 mixture at doses of 100,200 , and $400 \mathrm{mg} / \mathrm{kg}$ significantly reduced serum uric acid levels by $21 \%, 20 \%$, and $39 \%$, respectively, compared with the PO group $(p<$ 0.05, $p<0.05$, and $p<0.01$, resp.). Additionally, $10 \mathrm{mg} / \mathrm{kg}$ AP treatment decreased serum uric acid levels by $66 \%$ (Figure 2(a)). Urine uric acid levels were reduced in the PO group compared with the NC group $(p<$ $0.001)$. In contrast, treatment with $400 \mathrm{mg} / \mathrm{kg}$ CC12 mixture significantly increased urine uric acid levels by 1.6-fold $(p<$ 0.05). $10 \mathrm{mg} / \mathrm{kg}$ AP treatment did not affect urine uric acid levels (Figure 2(b)). FEUA was lower in the PO group than in the NC group. CC12 mixtures showed the tendency to increase the FEUA and $400 \mathrm{mg} / \mathrm{kg} \mathrm{CC12}$ mixture treatment significantly increased by 5 -fold compared with the PO group $(p<0.05$, Figure $2(c))$, although there was no significant change in serum and urine creatinine levels among treated groups (data not shown). $10 \mathrm{mg} / \mathrm{kg}$ AP also increased the FEUA $(p<0.05$, Figure 2(c)). of Its Components. HPLC chromatograms of $\mathrm{CF}, \mathrm{CB}$, and
TABLE 1: Contents of marker compounds in CC12 mixture.

\begin{tabular}{lccc}
\hline Contents (\%) & CF & CB & CC12 mixture \\
\hline Chlorogenic acid & 1.46 & & 0.55 \\
3,4-Dicaffeoylquinic acid & 0.46 & & 0.16 \\
Coumarin & & 4.24 & 3.70 \\
Cinnamaldehyde & & 15.13 & 11.84 \\
trans-Cinnamic acid & & 0.38 & 0.33 \\
$o$-Methoxycinnamaldehyde & & 1.1 & 1.55 \\
\hline
\end{tabular}

CF, Chrysanthemum indicum flower ethanol extract; CB, Cinnamomum cassia bark ethanol extract; $\mathrm{CC}$, mixture of $\mathrm{CF}$ and $\mathrm{CB}$.

the CC12 mixture are shown in Figure 3. Their chemical components were identified from UV spectra and retention times compared with reference materials. Four compounds, coumarin, cinnamaldehyde, trans-cinnamic acid, and $o$-methoxycinnamaldehyde, composed $\mathrm{CB}$, and two compounds, chlorogenic acid and 3,4-dicaffeoylquinic acid (isochlorogenic acid), composed CF. The representative HPLC chromatogram of the CC12 mixture showed three major components, chlorogenic acid, coumarin, and cinnamaldehyde, and their contents were $0.55,3.70$, and $11.84 \%$, respectively, as shown in Table 1 .

3.4. Effects of the CC12 Mixture and Its Components on XOD Activity In Vitro. Table 2 shows the effects of the CC12 mixture and its components on XOD activity. CF inhibited XOD activity by $39.2 \%$ at a concentration of $500 \mu \mathrm{g} / \mathrm{ml}$, showing weaker effects than its counterparts. CB inhibited XOD activity by $31.2 \%, 100.3 \%$, and $98.4 \%$, respectively, at concentrations of 100,250 , and $500 \mu \mathrm{g} / \mathrm{ml}$. The CC12 mixture also inhibited XOD activity at concentrations of 100, 250, and $500 \mu \mathrm{g} / \mathrm{ml}$ by $23.2,94.3$, and $101.0 \%$, respectively. We next investigated the components of $\mathrm{CF}$ and $\mathrm{CB}$. The $\mathrm{IC}_{50}$ values of chlorogenic acid and 3,4-dicaffeoylquinic acid (CF) were $103.8 \mu \mathrm{g} / \mathrm{ml}$ and $79.7 \mu \mathrm{g} / \mathrm{ml}$, respectively. The $\mathrm{IC}_{50}$ values of coumarin, cinnamaldehyde, trans-cinnamic acid, and $o$ methoxycinnamaldehyde (CB) were $95.2 \mu \mathrm{g} / \mathrm{ml}, 43.7 \mu \mathrm{g} / \mathrm{ml}$, $58.8 \mu \mathrm{g} / \mathrm{ml}$, and $25.7 \mu \mathrm{g} / \mathrm{ml}$, respectively.

\section{Discussion}

Hyperuricemia is a metabolic disorder characterized by an imbalance between uric acid production and excretion and causes gout. The prevalence of hyperuricemia has increased worldwide in recent decades [27]. Currently, only limited numbers of drugs are available for the treatment of hyperuricemia, and many of these agents have adverse effects. Therefore, more effective antihyperuricemia agents are needed.

In this study, we examined the effects of $\mathrm{CF}, \mathrm{CB}$, and their combination on hyperuricemia. We found that $\mathrm{CF}, \mathrm{CB}$, and various $\mathrm{CC}$ mixtures significantly decreased serum uric acid levels in PO-induced hyperuricemic rats. Our results correspond to those of previous reports demonstrating that Chrysanthemum flower oil and Cinnamomum bark and leaf oils reduced serum uric acid levels [20, 22, 23]. We 


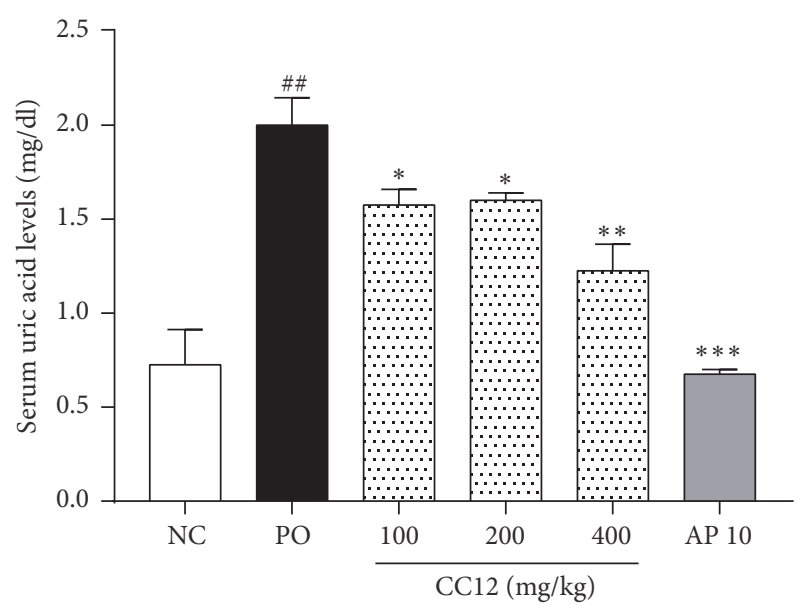

(a)

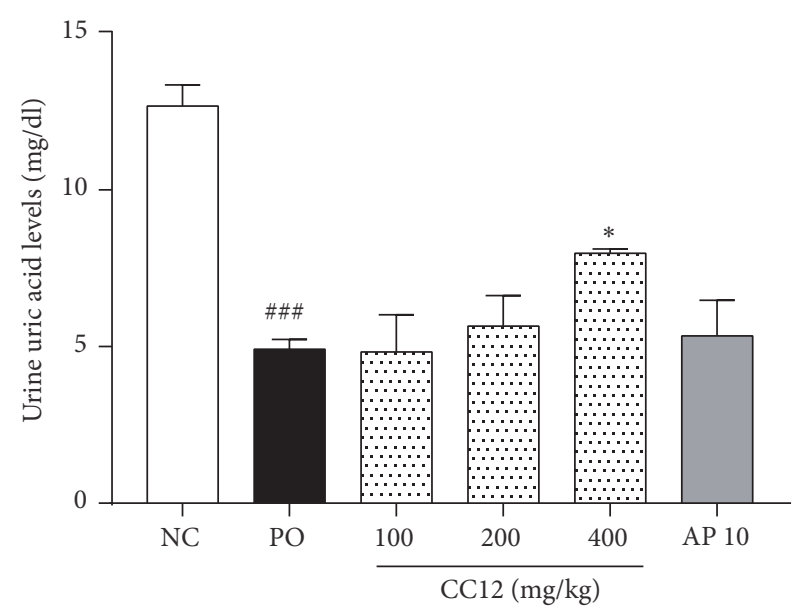

(b)

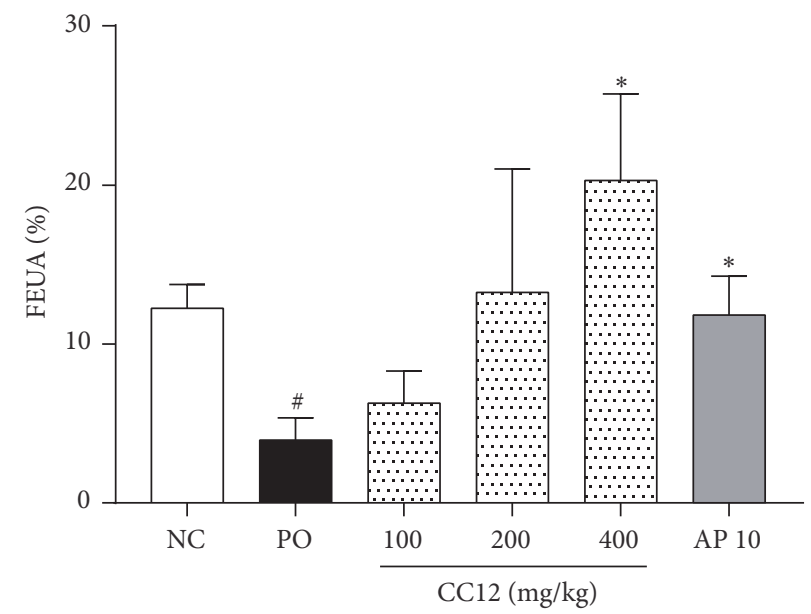

(c)

FIGURE 2: Dose-dependent effects of the CC12 mixture on serum (a), urine (b), uric acid levels, and fractional excretion of uric acid (FEUA) (c) in PO-induced hyperuricemic rat. NC, normal control group; PO, potassium oxonate-induced hyperuricemia group; CC12, mixture of $\mathrm{CF}$ and $\mathrm{CB}$ at a ratio of $1: 2$; AP $10,10 \mathrm{mg} / \mathrm{kg}$ allopurinol. Data are expressed as the mean $\pm \mathrm{SEM}(n=8) .{ }^{\# \#} p<0.01$ and ${ }^{\# \# \#} p<0.01$ versus the NC group; ${ }^{*} p<0.05,{ }^{* *} p<0.01$, and ${ }^{* * *} p<0.01$ versus the PO group.

also determined whether these agents exert allopurinol-like effects in normal rats. The CC mixtures reduced serum uric acid levels, while CF and CB did not affect uric acid levels in normal rats, indicating that CC mixtures can exert allopurinol-like hypouricemic effects. Moreover, these CC mixtures were more effective at decreasing serum uric acid levels than $\mathrm{CF}$ or $\mathrm{CB}$ alone, and the CC12 mixture exhibited the synergistic effects on serum uric acid levels in POinduced hyperuricemic rats and normal rats. Additionally, the $\mathrm{CC} 12$ mixture significantly reduced uric acid levels in a dose-dependent manner in PO-induced hyperuricemic rats, although its antihyperuricemic effects appeared to be weaker than those of allopurinol. These results suggest that the combination of CF and CB can exert enhanced antihyperuricemic effects. As mentioned above, although there have been reports regarding the antihyperuricemic effects of $\mathrm{CF}$ and $\mathrm{CB}$, this study is the first to demonstrate that a CC mixture, a combination of $\mathrm{CF}$ and $\mathrm{CB}$, exerts synergistic antihyperuricemic effects. These results suggest that the CC mixtures, as well as $\mathrm{CF}$ and $\mathrm{CB}$, exert hypouricemic effects and $\mathrm{CC} 12$ mixture exerts better antihyperuricemic effects than other CC mixtures, which may represent alternative treatments for hyperuricemia.

To elucidate the underlying mechanisms of the antihyperuricemic effects of the CC12 mixture, we evaluated the effects of the CC12 mixture on XOD activity. XOD is a limiting enzyme that catalyzes the oxidation of hypoxanthine to produce xanthine and the subsequent oxidation of xanthine to produce uric acid in purine catabolism [28, 29]. Because hyperuricemia results from uric acid overproduction and underexcretion, XOD inhibitors that can inhibit the production of uric acid have been considered for the treatment of hyperuricemia. Furthermore, it has been demonstrated that oils obtained from $\mathrm{CF}$ and $\mathrm{CB}$ can inhibit $\mathrm{XOD}$ in vitro $[21,23,30,31]$. In our study, we found that the CC12 mixture showed the tendency to inhibit the liver XOD activity in PO-induced hyperuricemic rats, although this effect was not significant (data not shown). CB and CC12 exerted 


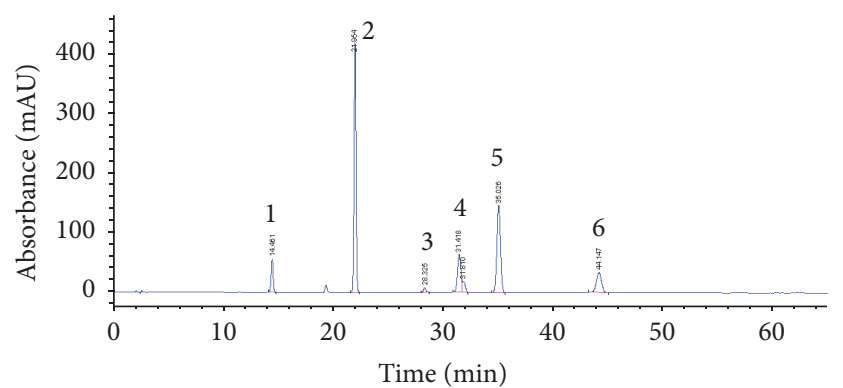

(a)

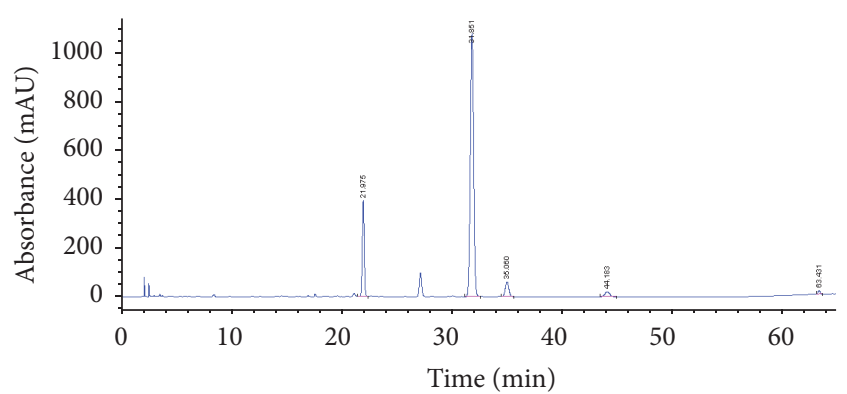

(c)

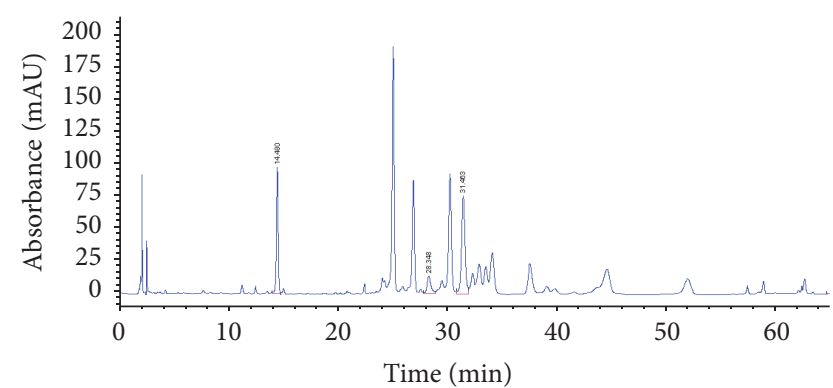

(b)

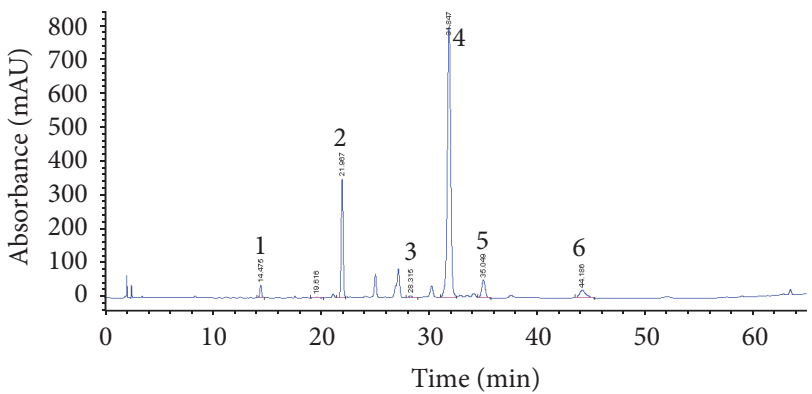

(d)

FiguRE 3: Representative HPLC chromatograms of a standard solution (a), CF (b), CB (c), and the CC12 mixture (d): (1) chlorogenic acid, (2) coumarin, (3) 3,4-dicaffeoylquinic acid, (4) cinnamaldehyde, (5) trans-cinnamic acid, and (6) o-methoxycinnamaldehyde.

moderate inhibitory effects on XOD in vitro, while CF exerted very weak inhibitory effects. In addition, 6 components of the CC12 mixture, namely, chlorogenic acid and 3,4dicaffeoylquinic acid from $\mathrm{CF}$ and coumarin, cinnamaldehyde, trans-cinnamic acid, and $o$-methoxycinnamaldehyde from $\mathrm{CB}$, exerted inhibitory effects on XOD activity in vitro. According to Wang et al. [23] and Meng et al. [32], chlorogenic acid and cinnamaldehyde significantly decreased serum uric acid levels by inhibiting XOD activity in POinduced hyperuricemic mice. Based on our results and previous reports, cinnamaldehyde may be the primary contributor to the inhibitory effects of $\mathrm{CB}$ and the $\mathrm{CC} 12$ mixture on $\mathrm{XOD}$ activity, as it is main constituent of these compounds (15.13\% and $11.84 \%$, resp.). In contrast, chlorogenic acid does not seem to be a major active compound because its content is too low $(1.46 \%)$, and CF exerted weaker inhibitory effects on XOD in vitro than its counterpart. In spite of its weaker inhibitory effects on XOD activity, CF was as effective at lowering serum uric acid levels as CB in PO-induced hyperuricemic rats, indicating that other mechanisms may underlie the hypouricemic effects of CF. Moreover, given that the CC12 mixture exerted relatively weaker inhibitory effects on XOD in vitro and strong uric acid-lowering effects in vivo, the synergistic effects of the CC12 mixture in vivo may also be attributable to other mechanisms. Thus, the beneficial antihyperuricemic effects exerted by the $\mathrm{CC} 12$ mixture in this study may be accomplished, at least in part, by its inhibitory effects on XOD, and its components may be responsible for its hypouricemic effects. However, further investigation is needed to elucidate the other mechanisms underlying the synergetic effects of the mixture.
Uric acid excretion as well as uric acid production plays an important role in uric acid homeostasis [2,3]. We further examined whether the CC12 mixture could increase renal uric acid excretion via analysis of urine uric acid levels and FEUA, renal uric acid handling parameter. FEUA is used as marker of proximal tubular handling of uric acid and is defined as the percentage of uric acid excreted to creatinine excreted by glomeruli which is finally excreted in the urine. Therefore, increased FEUA can be interpreted as the increased excretion of renal uric acid. In the present study, uric acid levels in urine and FEUA reduced in the PO group compared to the NC group although there are reports demonstrating the uricosuric effect of $\mathrm{PO}[33,34]$. This observation is in agreement with finding of various reports $[33,34]$. This discrepancy might be associated with the differences in approaches for administration periods and collecting time of urine. Interestingly, high doses of the CC12 mixture significantly increased urine uric acid levels and FEUA in PO-induced hyperuricemic rats. Previous report demonstrated that Chrysanthemum flower oil accelerated the uric acid excretion by upregulation of genes expression for uric acid transporters in kidney [22]. Based on this reporting, hypouricemic effect of $\mathrm{CF}$ might be related to enhanced uric acid excretion, which might be contributed to the synergistic effects of CC12 mixture. Our result suggests that CC12 mixture has ability to promote the renal uric acid excretion, which may be effect on reduced serum uric acid levels. However, additional studies are needed to analyze renal and urine uric acid levels of $\mathrm{CF}, \mathrm{CB}$, and their combination as well as the expression of renal organic anion transporters associated with urate handling. 
TABLE 2: XOD activity of CF, CB, and CC12 mixture and their marker compounds.

\begin{tabular}{|c|c|c|c|}
\hline Sample treatment & Concentration $(\mu \mathrm{g} / \mathrm{ml})$ & $\%$ inhibition & $\mathrm{IC}_{50}(\mu \mathrm{g} / \mathrm{ml})$ \\
\hline \multirow{3}{*}{ CF } & 100 & $5.3 \pm 2.8$ & \multirow{3}{*}{$>500$} \\
\hline & 250 & $8.9 \pm 4.1$ & \\
\hline & 500 & $39.2 \pm 6.9$ & \\
\hline \multirow{3}{*}{$\mathrm{CB}$} & 100 & $31.2 \pm 2.6$ & \multirow{3}{*}{143} \\
\hline & 250 & $100.3 \pm 3.1$ & \\
\hline & 500 & $98.4 \pm 0.1$ & \\
\hline \multirow{3}{*}{ CC12 mixture } & 100 & $23.2 \pm 0.4$ & \multirow{3}{*}{153.8} \\
\hline & 250 & $94.3 \pm 3.2$ & \\
\hline & 500 & $101.0 \pm 0.2$ & \\
\hline \multirow{3}{*}{ Chlorogenic acid } & 62.5 & $24.0 \pm 1.7$ & \multirow{3}{*}{103.8} \\
\hline & 125 & $63.6 \pm 1.5$ & \\
\hline & 250 & $107.3 \pm 0.6$ & \\
\hline \multirow{3}{*}{ 3,4-Dicaffeoylquinic acid } & 62.5 & $21.0 \pm 1.5$ & \multirow{3}{*}{79.7} \\
\hline & 125 & $104.6 \pm 2.6$ & \\
\hline & 250 & $85.0 \pm 1.2$ & \\
\hline \multirow{3}{*}{ Coumarin } & 62.5 & $22.8 \pm 0.1$ & \multirow{3}{*}{95.2} \\
\hline & 125 & $75.1 \pm 1.0$ & \\
\hline & 250 & $103.9 \pm 0.7$ & \\
\hline \multirow{3}{*}{ Cinnamaldehyde } & 62.5 & $101.2 \pm 3.2$ & \multirow{3}{*}{43.7} \\
\hline & 125 & $126.3 \pm 5.6$ & \\
\hline & 250 & $118.8 \pm 35.1$ & \\
\hline \multirow{3}{*}{ trans-Cinnamic acid } & 62.5 & $54.3 \pm 0.7$ & \multirow{3}{*}{58.8} \\
\hline & 125 & $96.7 \pm 1.9$ & \\
\hline & 250 & $114.9 \pm 0.4$ & \\
\hline \multirow{3}{*}{$o$-Methoxycinnamaldehyde } & 62.5 & $104.7 \pm 0.2$ & \multirow{3}{*}{25.7} \\
\hline & 125 & $101.2 \pm 0.2$ & \\
\hline & 250 & $100.0 \pm 0.4$ & \\
\hline \multirow{3}{*}{ Allopurinol } & 6.25 & $51.7 \pm 0.7$ & \\
\hline & 12.5 & $71.7 \pm 0.1$ & \\
\hline & 25 & $80.3 \pm 0.3$ & \\
\hline
\end{tabular}

Chrysanthemum indicum Linne flower ethanol extract; CB, Cinnamomum cassia (L.) J. Persl bark ethanol extract; CC, mixture of CF and CB. Data are expressed as the mean \pm SEM.

\section{Conclusions}

The present study demonstrated that the CC12 mixture reduced serum uric acid levels in normal and $\mathrm{PO}$-induced hyperuricemic rats and that its effects were stronger than those of its components, $\mathrm{CF}$ and $\mathrm{CB}$, alone. Moreover, the CC12 mixture and its compounds inhibited XOD activity in vitro. Thus, the antihyperuricemic effects of the CC12 mixture may be due, at least in part, to its inhibitory effects on XOD activity, although further study is necessary to determine its effects on renal uric acid excretion. Based on these results, we propose that the CC12 mixture exerts beneficial effects on hyperuricemia and may be useful in the treatment of hyperuricemia.

\section{Disclosure}

An earlier version of this work will be presented as a physical poster at World Congress and Expo on Traditional \& Alternative Medicine in June 20, 2017.

\section{Conflicts of Interest}

The authors have no conflicts of interest to declare in regard to the manuscript.

\section{Acknowledgments}

This research was supported by the grants from the Korea Institute of Oriental Medicine (K16030 and K16720) funded by Korea government (Ministry of Science, ICT \& Future Planning).

\section{References}

[1] P. Boffetta, C. Nordenvall, O. Nyren, and W. Ye, "A prospective study of gout and cancer," European Journal of Cancer Prevention, vol. 18, no. 2, pp. 127-132, 2009.

[2] H. K. Choi, D. B. Mount, and A. M. Reginato, "American physiological, S., pathogenesis of gout," Annals of Internal Medicine, vol. 143, no. 7, pp. 499-516, 2005. 
[3] F. Haidari, M. R. Rashidi, S. A. Keshavarz, S. A. Mahboob, M. R. Eshraghian, and M. M. Shahi, "Effects of onion on serum uric acid levels and hepatic xanthine dehydrogenase/xanthine oxidase activities in hyperuricemic rats," Pakistan Journal of Biological Sciences, vol. 11, no. 14, pp. 1779-1784, 2008.

[4] J. Huang, S. Wang, M. Zhu, J. Chen, and X. Zhu, "Effects of genistein, apigenin, quercetin, rutin and astilbin on serum uric acid levels and xanthine oxidase activities in normal and hyperuricemic mice," Food and Chemical Toxicology, vol. 49, no. 9, pp. 1943-1947, 2011.

[5] R. Kumar, Darpan, S. Sharma, and R. Singh, "Xanthine oxidase inhibitors: a patent survey," Expert Opinion on Therapeutic Patents, vol. 21, no. 7, pp. 1071-1108, 2011.

[6] N. Dalbeth and A. So, "Hyperuricaemia and gout: State of the art and future perspectives," Annals of the Rheumatic Diseases, vol. 69, no. 10, pp. 1738-1743, 2010.

[7] A. Fukunari, K. Okamoto, T. Nishino et al., "Y-700 [1-[3-Cyano4-(2,2-dimethylpropoxy)phenyl]-1H-pyrazole-4-carboxylic acid]: a potent xanthine oxidoreductase inhibitor with hepatic excretion," Journal of Pharmacology and Experimental Therapeutics, vol. 311, no. 2, pp. 519-528, 2004.

[8] A. So and B. Thorens, "Uric acid transport and disease," The Journal of Clinical Investigation, vol. 120, no. 6, pp. 1791-1799, 2010.

[9] S. Ishibuchi, H. Morimoto, T. Oe et al., "Synthesis and structureactivity relationships of 1-phenylpyrazoles as xanthine oxidase inhibitors," Bioorganic and Medicinal Chemistry Letters, vol. 11, no. 7, pp. 879-882, 2001.

[10] V. F. Azevedo, P. G. Buiar, L. H. Giovanella, C. R. Severo, and M. Carvalho, "Allopurinol, benzbromarone, or a combination in treating patients with gout: analysis of a series of outpatients," International Journal of Rheumatology, vol. 2014, Article ID 263720, 2014.

[11] T. Bardin, "Current management of gout in patients unresponsive or allergic to allopurinol," Joint Bone Spine, vol. 71, no. 6, pp. 481-485, 2004.

[12] P. Pacher, A. Nivorozhkin, and C. Szabó, “Therapeutic effects of xanthine oxidase inhibitors: renaissance half a century after the discovery of allopurinol," Pharmacological Reviews, vol. 58, no. 1, pp. 87-114, 2006.

[13] S. Hao, C. Zhang, and H. Song, "Natural products improving hyperuricemia with hepatorenal dual effects," Evidence-Based Complementary and Alternative Medicine, vol. 2016, Article ID 7390504, 2016.

[14] W. Cheng, J. Li, T. You, and C. Hu, "Anti-inflammatory and immunomodulatory activities of the extracts from the inflorescence of Chrysanthemum indicum Linné," Journal of Ethnopharmacology, vol. 101, no. 1-3, pp. 334-337, 2005.

[15] H. Matsuda, T. Morikawa, I. Toguchida, S. Harima, and M. Yoshikawa, "Medicinal flowers. VI. Absolute stereostructures of two new flavanone glycosides and a phenylbutanoid glycoside from the flowers of Chrysanthemum indicum L.: their inhibitory activities for rat lens aldose reductase," Chemical \& Pharmaceutical Bulletin, vol. 50, no. 7, pp. 972-975, 2002.

[16] S. K. Rad, M. S. Kanthimathi, N. A. Malek, G. S. Lee, C. Y. Looi, and W. F. Wong, "Cinnamomum cassia suppresses caspase-9 through stimulation of AKT1 in MCF-7 cells but Not in MDAMB-231 cells," PLoS ONE, vol. 10, no. 12, Article ID e0145216, 2015.

[17] Y.-K. Shin, H.-U. Son, J.-M. Kim, J.-C. Heo, S.-H. Lee, and J.G. Kim, "Cinnamomum cassia bark produced by solid-state fermentation with Phellinus baumii has the potential to alleviate atopic dermatitis-related symptoms," International Journal of Molecular Medicine, vol. 35, no. 1, pp. 187-194, 2015.

[18] A. Khan, M. Safdar, M. M. Ali Khan, K. N. Khattak, and R. A. Anderson, "Cinnamon improves glucose and lipids of people with type 2 diabetes," Diabetes Care, vol. 26, no. 12, pp. 32153218, 2003.

[19] N. Sharma, P. Trikha, M. Athar, and S. Raisuddin, "Inhibition of benzo[a]pyrene-and cyclophoshamide-induced mutagenicity by Cinnamomum cassia," Mutation Research-Fundamental and Molecular Mechanisms of Mutagenesis, vol. 480-481, pp. 179-188, 2001.

[20] X. Zhao, J. X. Zhu, S. F. Mo, Y. Pan, and L. D. Kong, "Effects of cassia oil on serum and hepatic uric acid levels in oxonateinduced mice and xanthine dehydrogenase and xanthine oxidase activities in mouse liver," Journal of Ethnopharmacology, vol. 103, no. 3, pp. 357-365, 2006.

[21] L. D. Kong, Y. Cai, W. W. Huang, C. H. K. Cheng, and R. X. Tan, "Inhibition of xanthine oxidase by some Chinese medicinal plants used to treat gout," Journal of Ethnopharmacology, vol. 73, no. 1-2, pp. 199-207, 2000.

[22] S. Honda, S. Kawamoto, H. Tanaka et al., "Administered chrysanthemum flower oil attenuates hyperuricemia: mechanism of action as revealed by DNA microarray analysis," Bioscience, Biotechnology and Biochemistry, vol. 78, no. 4, pp. 655-661, 2014.

[23] S. Y. Wang, C. W. Yang, J. W. Liao, W. W. Zhen, F. H. Chu, and S. T. Chang, "Essential oil from leaves of Cinnamomum osmophloeum acts as a xanthine oxidase inhibitor and reduces the serum uric acid levels in oxonate-induced mice," Phytomedicine, vol. 15, no. 11, pp. 940-945, 2008.

[24] J.-M. Li, X. Zhang, X. Wang, Y.-C. Xie, and L.-D. Kong, "Protective effects of cortex fraxini coumarines against oxonateinduced hyperuricemia and renal dysfunction in mice," European Journal of Pharmacology, vol. 666, no. 1-3, pp. 196-204, 2011.

[25] X. Wang, C.-P. Wang, Q.-H. Hu et al., "The dual actions of Sanmiao wan as a hypouricemic agent: down-regulation of hepatic XOD and renal mURAT1 in hyperuricemic mice," Journal of Ethnopharmacology, vol. 128, no. 1, pp. 107-115, 2010.

[26] S. H. Nile and S. W. Park, "Total phenolics, antioxidant and xanthine oxidase inhibitory activity of three colored onions (Allium cepa L.)," Frontiers in Life Science, vol. 7, no. 3-4, pp. 224-228, 2013.

[27] T. Shimo, N. Ashizawa, K. Matsumoto, T. Nakazawa, and O. Nagata, "Simultaneous treatment with citrate prevents nephropathy induced by FYX-051, a xanthine oxidoreductase inhibitor, in rats," Toxicological Sciences, vol. 87, no. 1, pp. 267276, 2005.

[28] I. A. Ramallo, S. A. Zacchino, and R. L. E. Furlan, "A rapid TLC autographic method for the detection of xanthine oxidase inhibitors and superoxide scavengers," Phytochemical Analysis, vol. 17, no. 1, pp. 15-19, 2006.

[29] T. Unno, A. Sugimoto, and T. Kakuda, "Xanthine oxidase inhibitors from the leaves of Lagerstroemia speciosa (L.) Pers," Journal of Ethnopharmacology, vol. 93, no. 2-3, pp. 391-395, 2004.

[30] C.-C. Lin, S.-J. Wu, C.-H. Chang, and L.-T. Ng, "Antioxidant activity of Cinnamomum cassia," Phytotherapy Research, vol. 17, no. 7, pp. 726-730, 2003.

[31] S. Sarawek, B. Feistel, I. Pischel, and V. Butterweck, "Flavonoids of Cynara scolymus possess potent xanthinoxidase inhibitory 
activity in vitro but are devoid of hypouricemic effects in rats after oral application," Planta Medica, vol. 74, no. 3, pp. 221-227, 2008.

[32] Z.-Q. Meng, Z.-H. Tang, Y.-X. Yan et al., "Study on the antigout activity of chlorogenic acid: improvement on hyperuricemia and gouty inflammation," American Journal of Chinese Medicine, vol. 42, no. 6, pp. 1471-1483, 2014.

[33] H. Yang, L. Gao, Y. Niu et al., "Mangiferin inhibits renal urate reabsorption by modulating urate transporters in experimental hyperuricemia," Biological and Pharmaceutical Bulletin, vol. 38, no. 10, pp. 1591-1598, 2015.

[34] Y. Yonetani, M. Ishii, and K. Iwaki, "Hyperuricemia induced by some antihypertensives and uricosuric drugs in oxonate-treated rats," Japanese Journal of Pharmacology, vol. 30, no. 6, pp. 829840, 1980. 


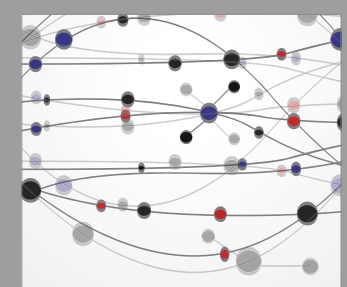

The Scientific World Journal
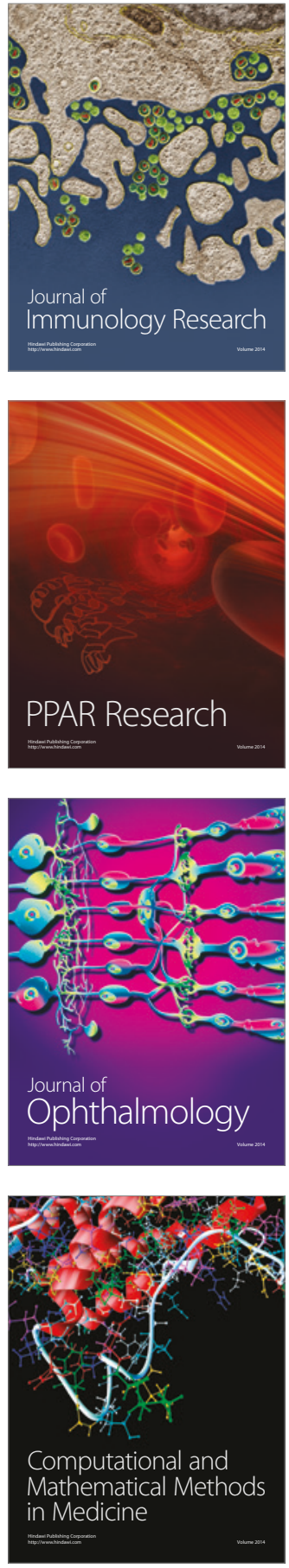

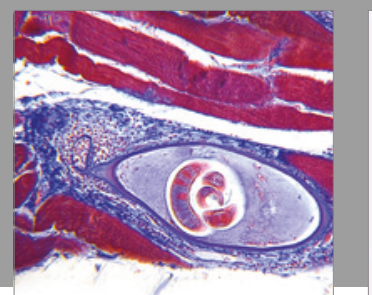

Gastroenterology Research and Practice
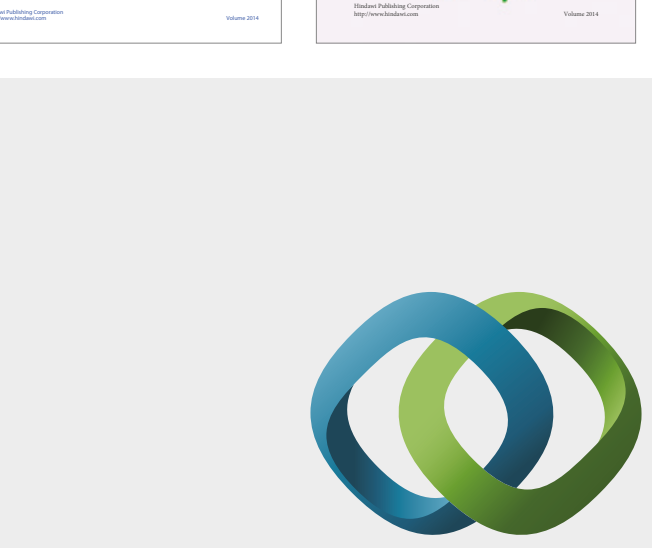

\section{Hindawi}

Submit your manuscripts at

https://www.hindawi.com
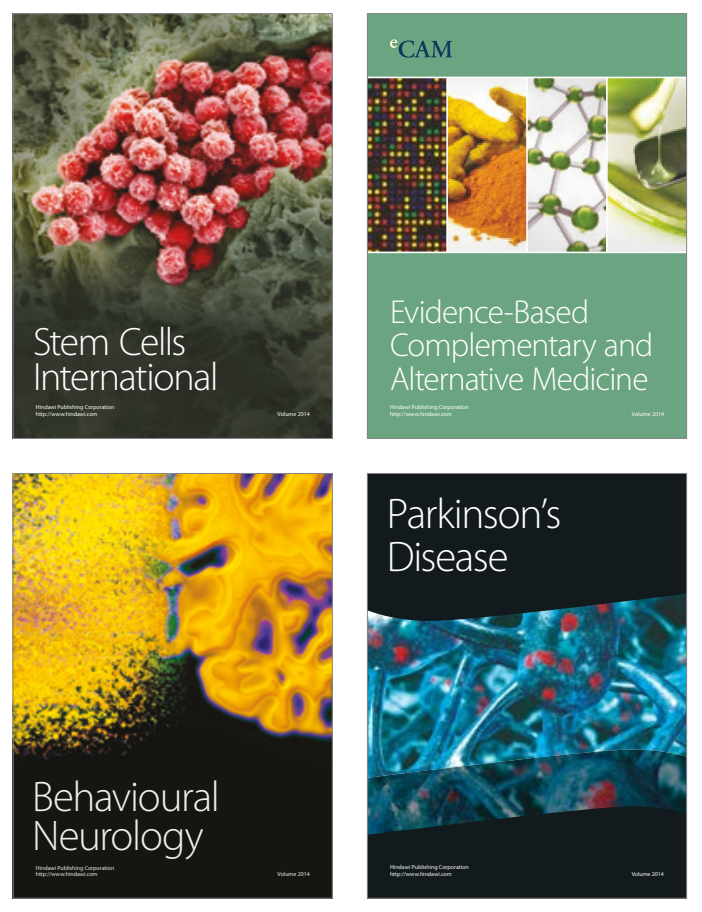
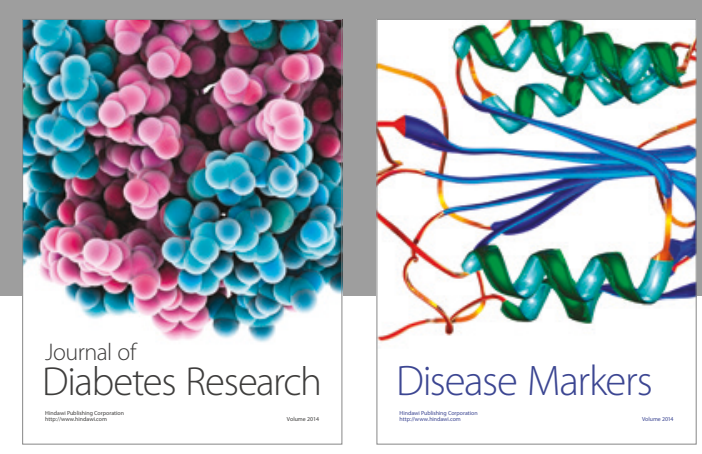

Disease Markers
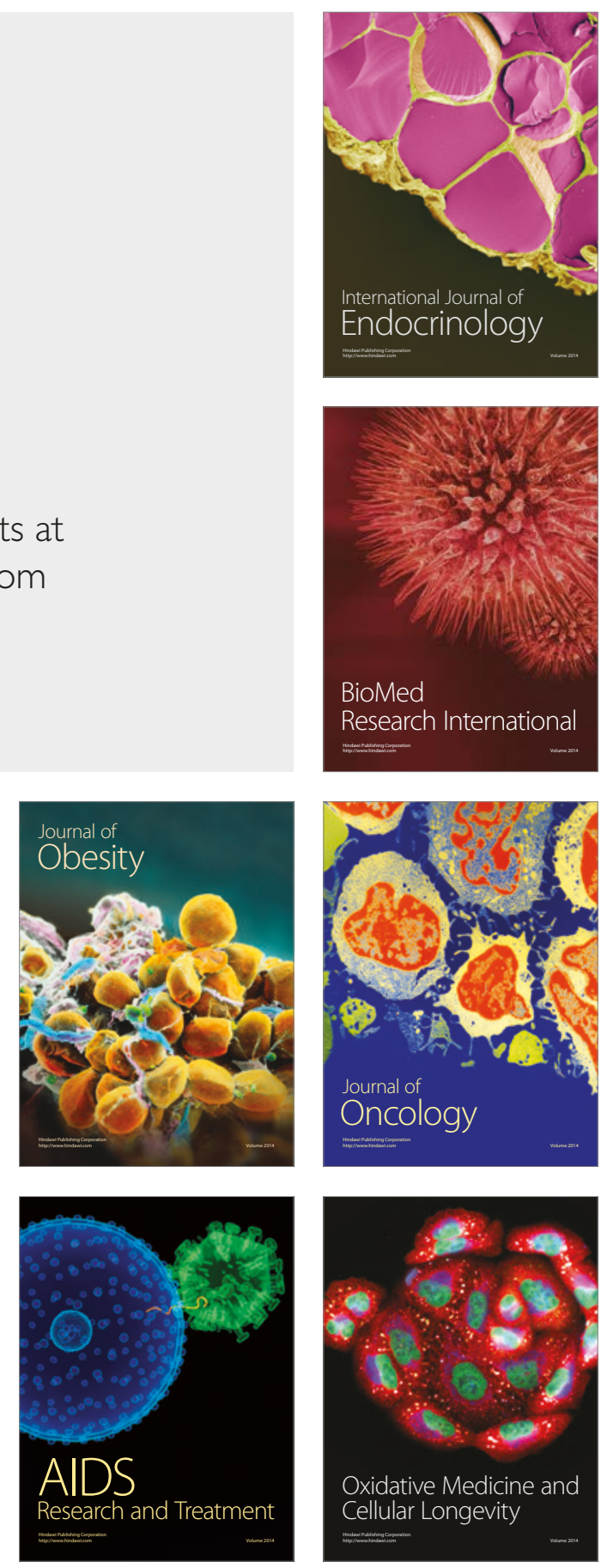